\title{
Aflatoxicosis in Animal Products and its Public Health Importance: A Review
}

\author{
Friat Kidanemariam ${ }^{1}$, Haben Fesseha ${ }^{2 *}$ \\ ${ }^{1}$ College of Veterinary Science, Mekelle University, P.O. Box: 2084, Mekelle, Ethiopia \\ ${ }^{2}$ School of Veterinary Medicine, Wolaita Sodo University, P.O. Box: 138, Wolaita Sodo, Ethiopia \\ *Corresponding Author: Haben Fesseha, School of Veterinary Medicine, Wolaita Sodo University, P.O. \\ Box: 138, Wolaita Sodo, Ethiopia
}

\begin{abstract}
Among all the mycotoxins, aflatoxins result in worldwide contamination of foods and feeds is a significant problem due to their extremely high toxicity and carcinogenicity effect on humans and animals. Aflatoxicosis is the poisoning that results from ingesting aflatoxins produced by the fungus Aspergillus species. It can cause direct animal losses, production losses, and trade limitations. However, the most alarming problem through time has been the presence of aflatoxin in milk, when eating contaminated forage all around the world. Aflatoxicosis has acute and chronic forms. Currently, aflatoxicosis has wider geographic distribution in animals and humans, however, the Incidence of aflatoxins in foods and feeds is relatively high in tropical and subtropical regions and this is mainly attributed to the risk factors physical, chemical and biological. All animal species are susceptible to this disease but the outbreak occurs mostly in pigs, sheep, and cattle. In animals, the toxin is processed through a number of competing pathways. Unfortunately, except for supportive therapy, there are almost no treatments for aflatoxin exposure. Prevention and control strategies for aflatoxicosis rely on biological control, testing feedstuffs, aflatoxin binders, and anti-fungal agents, blending down directing contaminated feed to least susceptible animals and Longer-term options include breeding for resistance in animals and breeding for lower susceptibility in crops.
\end{abstract}

Keywords: Aflatoxin, Feed, Human, Animal product, Public health

\section{INTRODUCTION}

Mycotoxins are low molecular weight secondary metabolites produced by certain strains of filamentous fungi such as Aspergillus, Penicillium, and Fusarium, which invade crops in the field and may grow on foods during storage under favorable conditions of temperature and humidity. They are regularly implicated in toxic syndromes in animals and humans (Charoenpornsook and Kavisarasai, 2006; Stasiewicz et al., 2017). The word aflatoxin is the combination of 3 words "a" for Aspergillus genus, "fla" for the species flavus and toxin, meaning poison (Atherstone et al., 2016; Bakırdere et al., 2012). Although aflatoxins have been a problem throughout history until 1960, they have been recognized as significant contaminants within agriculture (Unnevehr and Grace, 2013). Among all the mycotoxins, aflatoxins result in the greatest grain losses and highest management costs due to their extremely high toxicity (Asghar et al., 2016).

Livestock feeds are mainly composed of maize, groundnuts, soybean products, oil cake, fishmeal and brewers' grains, all of which are prone to contamination with aflatoxins (Pitt et al., 2012). Aflatoxin contaminates a large fraction of the world's food and other derivative products made from these primary feedstuffs in low-income countries (Caloni and Cortinovis, 2011). However, the most alarming problem through time has been the presence of aflatoxin-contaminated milk, because cows and goats (the major producers of drinking milk) are largely affected when eating contaminated forage all around the world (Lopez et al., 2003). Furthermore, Aflatoxin causes direct animal losses consequential morbidity and mortality, production losses and trade limitations, specifically in the international market, products that do not meet the aflatoxin standards are either rejected at the border, rejected in channels of distribution, assigned a reduced price, or diverted to nonhuman or even nonfee uses (Dhanasekaran et al., 2011). 
In animals, exposure of the fetus and via milk has been shown to have significant effects on the immune competence of progeny, even when maternal immunity factors were not influenced by the exposure (Dhanasekaran et al., 2011). Aflatoxin has a great impact on the decrease in immune globulins both in humans and in calves (Lizárraga-Paulín et al., 2011). As a result, it is also a concern with respect to HIV transmission to children and their ability to survive other infectious diseases (Ogbuewu, 2011; Williams et al., 2004).

Taking into consideration the importance of aflatoxicosis, the food and drug administration (FDA) has implemented regulations that required special attention to the management of the problem (FDAreport, 2010). However, the methods used to ensure minimal contamination in developed countries cannot realistically be used in developing countries, where these food systems and the technological infrastructure of the developing country are different from that of developed countries. As a consequence, the situation of aflatoxins in developing counties is in uncontrolled situations (Stasiewicz et al., 2017; Unnevehr and Grace, 2013) and there are no organized studies on the problem.

Yet few studies have examined aflatoxin contamination of feeds in East Africa. The limited information suggests a substantial proportion of feed is contaminated at levels likely to milk, egg, and poultry meat production. As livestock systems intensify, problems with aflatoxins are likely to worsen (Atherstone et al., 2016). Therefore, it is of paramount importance to study the magnitude, distribution, and overall epidemiology of the problem that leads to propose effective control measures. Thus, this paper was organized to review aflatoxicosis in animals and their public health importance.

\section{Aflatoxicosis In Animal Products}

\subsection{Cause and Sources}

Aflatoxicosis is the poisoning that results from ingesting aflatoxins and are produced by the fungus Aspergillus species and it occurs when animals ingest moldy corn, peanuts, or other foods susceptible to contamination by Aspergillus flavus or Aspergillus parasiticus (Cassel et al., 2001; Dhanasekaran et al., 2011). Aflatoxins produced by molds of Aspergillus species are of most interest because of acute toxicity and oncogenic effects in a susceptible host (Caloni and Cortinovis, 2011). Aflatoxins not only contaminate foods but also enter milk, eggs and livestock products through their feed (Alpsoy et al., 2009).

Eighteen types of aflatoxins have been identified, nevertheless, the naturally occurring and wellknown ones are aflatoxin B1 (AFB1), aflatoxin B2 (AFB2), aflatoxin G1 (AFG1) and aflatoxin G2 (AFG2) (Saleemullah et al., 2006). Aflatoxin M1 is a metabolite of aflatoxin B1 in humans and animals. Aflatoxin M2 is a metabolite of aflatoxin B1 in the milk of cattle fed on contaminated foods. Although aflatoxins B1, B2 and G1 are common in the same food sample, AFB1predominates (60$80 \%$ of the total aflatoxin content). Generally, AFB2, AFG1, and AFG2 don't occur in the absence of AFB1. Mostly, AFG1 is found in higher concentrations than AFB2 and AFG2 (Weidenborner, 2001).

Aflatoxins M1 and M2 (whose names are derived from milk aflatoxins, and then related to meat aflatoxins too), are thermo-resistant hydroxylated metabolites produced by lactating animals consuming aflatoxin-contaminated feeds. The ingested AFB1 and AFB2 are metabolized by livestock into AFM1 and AFM2, respectively, with an estimated conversion ratio of 1-3\% between AFB1 and AFM1 (Herzallah, 2009). Those toxic secondary metabolites named aflatoxins (AF) are a group of mycotoxins produced by a large number of Aspergillus species, basically by three phylogenetically distinct sections. The main producers are A. flavus, and A.parasiticus, but it has been demonstrated that A. nomius, A. pseudotamarii, A. parvisclerotigenus, and A.bombycis of section Flavi, A. ochraceoroseus and A. rambellii from section Ochraceorosei and Emericellaastellata and E. venezuelensis from Nidulatans section also generate aflatoxins (Frisvad et al., 2004).

Concentrated animal feedstuffs harbor the highest level of mycotoxins. For instance, the lowest level of aflatoxin B1 contamination recorded from silage feed, which is roughages, was $7 \mu \mathrm{g} / \mathrm{kg}$. However, the highest level of aflatoxin B1 contamination traced about $419 \mu \mathrm{g} / \mathrm{kg}$ in concentrate animal feeds like wheat bran, noug cake and sweat pea hull (De Boevre et al., 2012). Noug cake was warranted as the main source of aflatoxin contaminant among those concentrated animal feeds. Noug cake is increasingly used in Ethiopia for its high nutrient content to increase animal productivity in small scale or intensifying system. It is also exported to North America and Europe, where it is mainly used for bird feed (Gizachew et al., 2016). 


\subsection{Forms of Aflatoxicosis}

Aflatoxin can cause oncogenesis, chronic toxicity or per-acute signs depending on the species, age of animal, dose, and duration of aflatoxin exposure (Smith, 2002). Two forms of aflatoxicosis have been identified: the first is acute severe intoxication, which results in direct liver damage and subsequent illness or death, and the second is chronic sub symptomatic exposure (Jonathan et al., 2004).

\subsubsection{Acute Aflatoxicosis}

Acute aflatoxicosis is associated with high doses of aflatoxin (Wu et al., 2011; Probst et al., 2007). No animal species is immune to the acute toxic effects of aflatoxins (Williams et al., 2004). In 1960, more than 100,000 turkeys died in the United Kingdom over the course of a few months, prompting the name "Turkey X disease" (Asao et al., 1963). Later investigation revealed that the source of the disease was a toxic peanut meal. In 1981, several hundred calves that had been fed on peanut hay died in Australia (McKenize et al., 1981), and in 2007, several hundred animal deaths occurred on a chinchilla farm in Argentina; both these occurrences were linked to aflatoxin (González et al., 2008).

Moreover, in December 2005, several acute outbreaks of aflatoxicosis in dogs across the United States were raised more than 100 dogs became intoxicated and died within a few weeks (Stenske $e t$ al., 2006). Similarly, in December 2005, 2 dogs died of acute hepatic failure after consuming a commercially available food formulated for dogs (Bruchim et al., 2012; FDA-report, 2010). Grains for animal feed in the United States are allowed $300 \mathrm{ppb}$ aflatoxin because this concentration not only provides protection against acute aflatoxicosis but also is low enough to allow most of the grain produced to be traded. Overall acute aflatoxicosis is most likely underdiagnosed and under-reported (Liu and $\mathrm{Wu}, 2010$ ).

\subsubsection{Chronic Aflatoxicosis}

Chronic aflatoxin exposure has major effects on nutritional status in animals, but, as with the immune toxicities, thresholds for these effects are not defined for any species. Covalent binding of aflatoxin to DNA and decreased protein synthesis occur rapidly after exposure and persist for $\leq 5$ days (Williams et al., 2004). The efficiency of food use is consistently less in animals that are exposed to aflatoxin than in those that are not exposed (Lizárraga-Paulín et al., 2011).

In poultry and pigs, a 7-10\% drop in food conversion efficiency, and decreased growth rates are a consistent sign of chronic aflatoxin exposure. "Dose-related" decrease in weight gain in weaning piglets exposed to $140 \mathrm{ppb}$ and $280 \mathrm{ppb}$ aflatoxin has been documented (Ogbuewu, 2011). Chronic exposure to even low levels of contamination in crops consumed regularly increases liver, gallbladder cancer risk and can suppress the immune system (Nogueira et al., 2015; Stepman, 2018). However, in these animal feeding situations, the long-term risk of cancer is not a concern, except for the most susceptible species. Consequently, veterinary research has examined higher levels of exposure but for shorter time periods (Unnevehr and Grace, 2013).

\subsection{Epidemiology}

\subsubsection{Geographical Distribution}

The incidence of aflatoxins in foods and feeds is relatively high in tropical and subtropical regions, where climatic conditions favor the growth of molds (Liu and $\mathrm{Wu}, 2010)$. These areas have humid and dry climates that are highly suitable for the proliferation of fungal species, particularly Aspergillus flavus and Aspergillus parasiticus, which are the main producers of AFB1. Food-grains may be contaminated by AFB1 at a number of stages, but is most common when crops are exposed during harvesting and storage, provided that hot and humid weather conditions, improper and unsanitary storage exist for a prolonged period (Stepman, 2018; Williams et al., 2004).

\subsubsection{Species Involved}

All animal species including the honeybees are susceptible to aflatoxicosis, but the outbreak occurs mostly in pigs, sheep, and cattle (Atherstone et al., 2016). Poultries are more sensitive to aflatoxin than mammals. Within poultry, ducks are the most susceptible species followed by turkey and chickens. Within domestic animals, the order of susceptible, canine is the most susceptible species followed by swine, calves, cattle, and sheep. Young animals are more susceptible to AFs than adult animals (Bryden, 2011). Aflatoxicosis is typically a herd rather than an individual animal problem 
(Stasiewicz et al., 2017; Unnevehr and Grace, 2013). Recent findings suggest that wild animals may be substantially more susceptible to aflatoxicosis and it may be responsible for many idiopathic wildlife deaths (Pitt et al., 2012; Stepman, 2018).

\subsubsection{Risk Factors}

The formation of aflatoxins is influenced by physical, chemical and biological factors. The physical factors include temperature and moisture. The chemical factors include the composition of the air and the nature of the substrate. Biological factors are those associated with the host species (Feddern et al., 2013). Levels of humidity, temperature, and aeration during storage are also important factors that are intimately related to the actual problems of climate change and environmental warming around the whole world (Liu and $\mathrm{Wu}, 2010$ ). Synthesis of aflatoxins in feeds is increased at temperatures above $27^{\circ} \mathrm{C}$, humidity levels greater than $62 \%$ and moisture levels in the feed above $14 \%$ (Mutegi et al., 2018)).

The main predisposing factor in postharvest aflatoxin accumulation in food is poor storage conditions; namely, excessive heat and moisture, pest-related crop damage, and extensive periods of time spent in storage (exceeding several months) (Neme and Mohammed, 2017). Even commodities dried to a satisfactory degree before storage can develop local pockets favorable to aflatoxin growth as a result of moisture generated by insect respiration and local condensation (Betrán and Isakeit, 2004; Dohlman, 2003). Genotypes, drought, soil types and insect activity are important in determining the likelihood of pre-harvest contamination. All these factors increase the risk of the crop plant being infected by A. flavus or A. parasiticus (Mutegi et al., 2018; Neme and Mohammed, 2017; Wolde, 2017).

Expression of aflatoxin-related diseases is influenced by factors such as species, age, nutrition, sex, and the possibility of concurrent exposure to other toxins (Neme and Mohammed, 2017). Sex and age of animals have also an influence on AFB1 susceptibility. For instance, males are more susceptible than females and young animals of all species are more susceptible than mature animals to the effects of aflatoxin. Nutritional deficiencies, especially protein and vitamin E increase the susceptibility to AFs (Bryden, 2011; Stasiewicz et al., 2017).

\subsection{Pathogenesis}

In animals, the toxin is processed through a number of competing pathways (Feddern et al., 2013). Animal feeds are an essential part of the farm animal to the human food chain. So, Aflatoxins are not only present in milk and derivatives even after pasteurizing processes and heating, but there have also been found in other food animal products like turkey and hen eggs and meat are the source of aflatoxin contamination in human foods. The Cytochrome p450 (CYP) enzymes are a superfamily of hemeproteins that are important in the oxidative, peroxidative and reductive metabolism of endogenous compounds and participate in the chemical carcinogenesis process (Lizárraga-Paulín et al., 2011).

Aflatoxin is activated by CYP1A2 and CYP3A4 to $\mathrm{AFB}_{1}-8$, 9-epoxide is a highly unstable molecule that has a high affinity for guanine $(\mathrm{G})$ bases in the DNA, which covalently binds with DNA to form DNA-adducts, primarily $\mathrm{AFB}_{1}$-N7-guanine in the target cells. These adducts induce DNA mutations primarily through a $\mathrm{G}$ (guanine) to $\mathrm{T}$ (thymine) transversion in the DNA. Thus, transversion (AGG to AGT) at the third base of codon 249 of the protein 53 (p53) tumor suppressor genes in the human or animal liver induces changes in the coding properties of the DNA (Guengerich et al., 1998).

\subsection{Toxicity and Clinical Signs}

When aflatoxins are consumed, it can exert toxicity in several ways. It may alter intestinal integrity or modulate the expression of cytokines, proteins that "signal" to each other and to immune system components (Gong et al., 2008; Leslie et al., 2008). The high toxicity and carcinogenicity of these compounds and their ability to cause various pathological conditions has led to the widespread screening of foods and feeds potentially contaminated with them. Furthermore, in some studies on pregnant hamsters showed a significant relationship between exposure of aflatoxin B1 $(4 \mathrm{mg} / \mathrm{kg}$, single dose) and the appearance of developmental anomalies in their offspring (Goldblatt, 2012; Wolde, 2017). 
The adverse effects of exposure to aflatoxin on various indicators of growth performance have been demonstrated in multiple animal species (Dohlman, 2003). Across all species provides clear evidence that the dose and duration of exposure to aflatoxin clearly have a major effect on the toxicology and may cause a range of consequences: 1) large doses lead to acute illness and death, usually through liver cirrhosis; 2) chronic sub-lethal doses have nutritional and immunologic consequences; and 3) all doses have a cumulative effect on the risk of cancer (Jonathan et al., 2004). However, the differences between species in response to aflatoxin introduce a measure of speculation into the extension of farm animal-derived information to the human situation (Williams et al., 2004).

The main target organ in mammals is the liver, so aflatoxicosis primarily is a hepatic disease (Lizárraga-Paulín et al., 2011). Aflatoxins have been cause to liver damage, kidney disorders, gastrointestinal dysfunction, reduced productivity, decreased feed utilization and efficiency, decreased reproductive performance (including reduced hatchability, smaller eggs, and reduced eggshell quality), reduced milk or egg production, embryonic death, teratogenicity (birth defects), tumors, and suppressed immune system function, even when low levels are consumed (Ogbuewu, 2011). Aflatoxin B1, aflatoxin M1, and aflatoxin G1 have been shown to cause various types of cancer in different animal species. However, only aflatoxin B1 is considered as having produced sufficient evidence of carcinogenicity in experimental animals to be identified as a carcinogen (Bennett and Klich, 2003).

\subsection{Diagnosis}

Diagnosis of aflatoxicosis is often difficult because of the variation in clinical signs, gross pathological conditions and the presence of infectious diseases due to the suppression of the immune system. In addition, the farm, more than one mold or toxin may be present in the contaminated feed, which often makes a definitive diagnosis of aflatoxicosis difficult. The prognosis of aflatoxicosis depends upon the severity of liver damage (Stasiewicz et al., 2017). Despite this, a presumptive diagnosis can be based on historical exposure to contaminated feed, presence of aflatoxins in food sources, vomitus, intestinal contents characteristic, histological findings and microscopic examination of the liver (Neme and Mohammed, 2017).

Chronic aflatoxicosis may be diagnosed by determining serum biochemical and hematological alterations before clinical symptoms become apparent. Acutely affected animals have increased in liver enzymes (alkaline phosphatase, AST, or ALT), bilirubin, serum bile acids, and prothrombin time (Feddern et al., 2013).

\subsection{Treatment}

As it has been mentioned before, most aflatoxicosis results from eating contaminated feeds. Unfortunately, except for supportive therapy (e.g., diet and hydration), there are almost no treatments for aflatoxin exposure (Lizárraga-Paulín et al., 2011; Probst et al., 2007). For hepatic damage caused by aflatoxins, treatments include S-adenosylmethionine, milk thistle, vitamin $\mathrm{K} 1$, vitamin $\mathrm{E}$, nutritional support, and fresh-frozen plasma (Stenske et al., 2006). Milk thistle (Silybummarianum), which is a medicinal herb found in Pakistan, has been used to treat liver diseases. This herb was tested in poultry feed contaminated with AFB1 ( $80 \mathrm{ppb}$ for the first week and $520 \mathrm{ppb}$ from the second until the fifth) at a dose of $1 \%$. The results indicated that milk thistle is effective as hepatoprotection and growth promoter in the presence of AFB1 in the feed (Muhammad et al., 2012; Stepman, 2018).

\subsection{Prevention and Control}

Removing and control of aflatoxin from contaminated feeds and foodstuffs remains a major problem, and there is a great demand for effective decontamination technology (Leslie et al., 2008). There are several technical options including biological control, testing feedstuffs, aflatoxin binders, and antifungal agents, blending down and directing contaminated feed to least susceptible animals. Longerterm options include breeding for resistance in animals and breeding for lower susceptibility in crops (Unnevehr and Grace, 2013). The use of adsorbents (silicates) or chemicals (ammonia, sodium bisulfate) or biological agents (Saccharomyces cerevisiae) in animal feeds has been shown to minimize the effect of aflatoxin. Esterified glucomannan (EG) showed a very high binding ability (8097\%) with aflatoxin (Basmacioglu et al., 2005). It consists of principally in good agricultural practice, 
including an appropriate drying of crops after harvest and avoidance of moisture during storage (Lewis et al., 2005; Lizárraga-Paulín et al., 2011).

Adsorbents are necessary and important and may have a great impact on improving animal production and health. One of the promising prospects is the addition of dietary clay to dry diets during the extrusion process or as a coating on the surface of the kibble (Bingham et al., 2004). Clay derived sorbents is type of binder that is basically composed of single or blended types of clay. The most common clay is hydrated sodium calcium aluminosilicate (HSCAS). However, there are other sorts of clays that can be used as toxin binders, like sodium or calcium bentonites and zeolites (Feddern et al., 2013). In addition, soil treatment with nontoxigenic strains had the beneficial carry-over effect of reducing aflatoxin contamination that occurred during storage (Dorner and Cole, 2002).

The most well-known natural toxin binders are yeast-based products. Glucans are yeast cell wall constituents. Those compounds have been submitted to the esterification process generating a new additive with toxin binding capacity, called esterified glucomannan (EGM) (Aravind et al., 2003). Moreover, the use of probiotics that improve animal health and physiology can be helpful. Plant extracts with a specific mode of action, like liver protection, have been used as well to reduce the toxicity of some mycotoxins, especially aflatoxin (Feddern et al., 2013).

The traditional approach to preventing exposure to aflatoxin has been to ensure that foods consumed have the lowest practical aflatoxin concentrations. In developed countries, where regulations allow higher aflatoxin concentrations in animals, the agricultural industries have developed alternative approaches chemoprotection and enterosorption to limit biologically effective exposure without the high cost of preventing contamination (Galvano et al., 2001; Wolde, 2017). Chemoprotection is based on manipulating the biochemical processing of aflatoxin to ensure detoxification rather than preventing biological exposure. Enterosorption is based on the approach of adding a binding agent to food to prevent the absorption of the toxin while the food is in the digestive tract; the combined toxinsorbent is then excreted in the feces. This approach has been used extensively and with great success in the animal feeding industry (Mutegi et al., 2018; Pitt et al., 2012).

\section{Public Health Significance}

Aflatoxicosis is the third-leading cause of cancer death globally, with about 550,000-600,000 new cases each year and the highest incidence of 40 percent is in Africa (Liu and Wu, 2010). Eighty-three percent of these deaths occur in East Asia and sub-Saharan Africa (Mutegi et al., 2018; Stepman, 2018). Animal feeds are an essential part of the farm animal to the human food chain; therefore, infectious and noninfectious hazards present in animal feeds pose a threat to human health (LizárragaPaulín et al., 2011). So, Aflatoxins are not only present in cow, goat and sheep milk and derivatives even after pasteurizing processes, there have also been found in other food animal products like turkey and hen eggs. Residues of aflatoxins and their metabolites in foodstuff animal tissues (like beef and sheep meat) may be a source of aflatoxin contamination in human foods. Developing countries located in tropical regions are at greatest risk given their reliance on these commodities as their staple food source (Neme and Mohammed, 2017).

Among the most potent hepatocarcinogenic agents known is aflatoxin. The major impetus arose from epidemiological evidence linking chronic aflatoxin exposure with the incidence of cancer in humans. It is secreted in the milk and it is highly stable; heating will not break down the toxin sufficiently. Subsequently, the toxins are further processed into yogurt, cheese, and butter. This means that milk and other dairy product pose a threat to humans, particularly children more vulnerable because milk consumption is often higher among infants and children. Consumption of milk (products) suppresses their immunity and contributes to stunting (Asghar et al., 2016; Gizachew et al., 2016).

In Africa, the connection between infectious diseases and aflatoxin is also reported for malaria, and some interesting effects are noted. The most of the information on the toxicities of aflatoxin at intermediate rates of exposure (100-500 ppb) and is the most potentially relevant information that is appropriate for the human situation in developing countries where no control of aflatoxin is exercised (Williams et al., 2004). AFM1 levels in the samples in Khartoum state appear to be a serious public health problem. AFM1 contamination in the samples of dairy cattle milk in Khartoum state appears to be prevalent and may pose a public health problem at the moment (Suliman and Abdalla, 2013). As of 2014, the most recent outbreak of more than one case from a single food source occurred in rural Kenya in 2004-2005, when 317 people became sick and 125 died of acute aflatoxicosis indicate a case fatality rate as high as 60\% (Asghar et al., 2016; Mutegi et al., 2018). 
The expression of aflatoxin-related diseases in humans may be influenced by factors such as age, sex, nutritional status, to underweight status in children and/or concurrent exposure to other causative agents such as viral hepatitis (HBV) or parasite infestation (Bennett and Klich, 2003; Berek et al., 2001). In addition, aflatoxin exposure and the toxic effects on immunity and nutrition combine to negatively affect health factors (including HIV infection) that account for $>40 \%$ of the burden of disease in developing countries where a short lifespan is prevalent (Pitt et al., 2012; Stepman, 2018; Unnevehr and Grace, 2013)

Exposure to aflatoxins been implicated in hepatocellular carcinoma, hepatic failure, encephalopathy, and Reye's syndrome, such exposure may also be important in the health and well-being of the fetus and neonates (Bennett and Klich, 2003). Understanding the interaction of viral infection, genetic variation and exposure to environmental chemical carcinogens will help to elucidate mechanisms of human hepatocarcinogenesis and develop more effective strategies for HCC prevention (Zhang, 2010).

\section{CONCLUSION AND RECOMMENDATIONS}

Aflatoxicosis is often difficult to diagnosis and treatment. In spite of different research on aflatoxins, several areas of aflatoxicosis remain yet to be explored. In East Africa livestock some farmers are known to channel worst quality maize use to animal feed. While a great deal is known about aflatoxins, it is not much known about aflatoxin exposure and the resulting health effects in developing countries. The economic impact of the disease in developing countries is not only limited to the high cost of production lost but also the cost lost from international trade. In addition to their intrinsic scientific and medical significance, aflatoxicosis has also assumed tremendous public health importance. Recent findings suggest that wild individuals may be substantially more susceptible to aflatoxicosis and it may be responsible for many idiopathic wildlife deaths. Removing and control of aflatoxins from contaminated feeds and foodstuffs remains a major problem. Even though the developed countries can control and prevention of aflatoxicosis has been achieved for humans and animals largely by regulations that have required low concentrations of the toxin in traded foods this approach has less promising in developing countries. In conclusion, awareness should be created for farm owners about the disease and animal feed management through community services. Researches on the mechanism of aflatoxin in animal products related to human health hazards should be carried out.

\section{REFERENCES}

[1] Aravind, K., Patil, V., Devegowda, G., Umakantha, B., and Ganpule, S. (2003). Efficacy of esterified glucomannan to counteract mycotoxicosis in naturally contaminated feed on performance and serum biochemical and hematological parameters in broilers. Poultry Science 82, 571-576.

[2] Asghar, M. A., Iqbal, J., Ahmed, A., Khan, M. A., Shamsuddin, Z. A., and Jamil, K. (2016). Development and validation of a high-performance liquid chromatography method with post-column derivatization for the detection of aflatoxins in cereals and grains. Toxicology and industrial health 32, 1122-1134.

[3] Atherstone, C., Grace, D., Lindahl, J. F., Kang'ethe, E. K., and Nelson, F. (2016). Assessing the impact of aflatoxin consumption on animal health and productivity. African Journal of Food, Agriculture, Nutrition and Development 16, 10949-10966.

[4] Bakırdere, S., Bora, S., Bakırdere, E. G., Aydın, F., Arslan, Y., Komesli, O. T., Aydın, I., and Yıldırım, E. (2012). Aflatoxin species: their health effects and determination methods in different foodstuffs. Central European journal of chemistry 10, 675-685.

[5] Basmacioglu, H., Oguz, H., Ergul, M., Col, R., and Birdane, Y. (2005). Effect of dietary esterified glucomannan on performance, serum biochemistry and haematology in broilers exposed to aflatoxin. Czech Journal of Animal Science 50, 31-39.

[6] Bennett, J., and Klich, M. (2003). Mycotoxins. Clinical Microbiological Reviews, 16.

[7] Berek, L., Petri, I. B., Mesterhazy, A., Téren, J., and Molnár, J. (2001). Effects of mycotoxins on human immune functions in vitro. Toxicology in vitro 15, 25-30.

[8] Betrán, F., and Isakeit, T. (2004). Aflatoxin accumulation in maize hybrids of different maturities. Agronomy Journal 96, 565-570.

[9] Bingham, A., Huebner, H., Phillips, T., and Bauer, J. (2004). Identification and reduction of urinary aflatoxin metabolites in dogs. Food and chemical toxicology 42, 1851-1858.

[10] Bruchim, Y., Segev, G., Sela, U., Bdolah-Abram, T., Salomon, A., and Aroch, I. (2012). Accidental fatal aflatoxicosis due to contaminated commercial diet in 50 dogs. Research in veterinary science 93, 279-287. 
[11] Bryden, W. (2011). Mycotoxins: Natural food chain contaminants and human health. Encyclopedia of Environmental Health, 898-905.

[12] Caloni, F., and Cortinovis, C. (2011). Toxicological effects of aflatoxins in horses. The veterinary journal 188, 270-273.

[13] Cassel, E. K., Campbell, B., Draper, M., and Epperson, B. (2001). Aflatoxins: Hazards in grain/aflatoxicosis and livestock. Fact Sheets, 86.

[14] Charoenpornsook, K., and Kavisarasai, P. (2006). Mycotoxins in animal feedstuffs of Thailand. KMITL Science and Technology Journal 6, 25-28.

[15] De Boevre, M., Di Mavungu, J. D., Landschoot, S., Audenaert, K., Eeckhout, M., Maene, P., Haesaert, G., and De Saeger, S. (2012). Natural occurrence of mycotoxins and their masked forms in food and feed products. World Mycotoxin Journal 5, 207-219.

[16] Dhanasekaran, D., Shanmugapriya, S., Thajuddin, N., and Panneerselvam, A. (2011). Aflatoxins and aflatoxicosis in human and animals. Aflatoxins-Biochemistry and Molecular Biology, 221-254.

[17] Dohlman, E. (2003). Mycotoxin hazards and regulations. International Trade and Food Safety, 97.

[18] Dorner, J. W., and Cole, R. J. (2002). Effect of application of nontoxigenic strains of Aspergillus flavus and A. parasiticus on subsequent aflatoxin contamination of peanuts in storage. Journal of Stored Products Research 38, 329-339.

[19] FDA-report (2010). FDA report on Diamond Pet Food Processors of South Carolina,LLC. Jan 19, 2006 Food and Drug Administration Available from: <http://www.fda.gov/ora/frequent/483s/01192006_DiamondPetFood/Diamond_Pet_Food_ProcessorsFDA 483_01192006.pdf>.

[20] Feddern, V., Dors, G. C., Tavernari, F. d. C., Mazzuco, H., Cunha, A., Krabbe, E. L., and Scheuermann, G. N. (2013). Aflatoxins importance on animal nutrition. Aflatoxins-Recent Advances and Future Prospects, 171-195.

[21] Galvano, F., Piva, A., Ritieni, A., and Galvano, G. (2001). Dietary strategies to counteract the effects of mycotoxins: a review. Journal of food protection 64, 120-131.

[22] Gizachew, D., Szonyi, B., Tegegne, A., Hanson, J., and Grace, D. (2016). Aflatoxin contamination of milk and dairy feeds in the Greater Addis Ababa milk shed, Ethiopia. Food control 59, 773-779.

[23] Goldblatt, L. (2012). "Aflatoxin: scientific background, control, and implications," Elsevier.

[24] Gong, Y. Y., Turner, P. C., Hall, A. J., and Wild, C. P. (2008). Aflatoxin exposure and impaired child growth in West Africa: an unexplored international public health burden. In "Mycotoxins: detection methods, management, public health and agricultural trade" (J. F. Leslie, ed.), pp. 53-65. CAB International.

[25] Guengerich, F. P., Johnson, W. W., Shimada, T., Ueng, Y.-F., Yamazaki, H., and Langouët, S. (1998). Activation and detoxication of aflatoxin B1. Mutation Research/Fundamental and Molecular Mechanisms of Mutagenesis 402, 121-128.

[26] Leslie, J. F., Bandyopadhyay, R., and Visconti, A. (2008). "Mycotoxins: detection methods, management, public health and agricultural trade," CABI.

[27] Lewis, L., Onsongo, M., Njapau, H., Schurz-Rogers, H., Luber, G., Kieszak, S., Nyamongo, J., Backer, L., Dahiye, A. M., and Misore, A. (2005). Aflatoxin contamination of commercial maize products during an outbreak of acute aflatoxicosis in eastern and central Kenya. Environmental health perspectives 113, 17631767.

[28] Liu, Y., and Wu, F. (2010). Global burden of aflatoxin-induced hepatocellular carcinoma: a risk assessment. Environmental health perspectives 118, 818-824.

[29] Lizárraga-Paulín, E. G., Moreno-Martínez, E., and Miranda-Castro, S. P. (2011). Aflatoxins and their impact on human and animal health: an emerging problem. Aflatoxins-Biochemistry and Molecular Biology 13, 255-282.

[30] Lopez, C., Ramos, L., Ramadan, S., and Bulacio, L. (2003). Presence of aflatoxin M1 in milk for human consumption in Argentina. Food control 14, 31-34.

[31] Muhammad, D., Chand, N., Khan, S., Sultan, A., and Mushtaq, M. (2012). Hepatoprotective Role of Milk Thistle (Silybum marianum) in Meat Type Chicken Fed Aflatoxin B 1 Contaminated Feed. Pakistan Veterinary Journal 32.

[32] Mutegi, C., Cotty, P., and Bandyopadhyay, R. (2018). Prevalence and mitigation of aflatoxins in Kenya (1960-to date). World Mycotoxin Journal 11, 341-357.

[33] Neme, K., and Mohammed, A. (2017). Mycotoxin occurrence in grains and the role of postharvest management as a mitigation strategies. A review. Food Control 78, 412-425. 
[34] Nogueira, L., Foerster, C., Groopman, J., Egner, P., Koshiol, J., and Ferreccio, C. (2015). Association of aflatoxin with gallbladder cancer in Chile. Jama 313, 2075-2077.

[35] Ogbuewu, I. (2011). Effects of mycotoxins in animal nutrition: a review. Asian J Anim Sci 5, 1933International.

[36] Pitt, J. I., Wild, C. P., Baan, R. A., Gelderblom, W. C., Miller, J., Riley, R., and Wu, F. (2012). "Improving public health through mycotoxin control," International Agency for Research on Cancer Lyon, France.

[37] Probst, C., Njapau, H., and Cotty, P. J. (2007). Outbreak of an acute aflatoxicosis in Kenya in 2004: identification of the causal agent. Appl. Environ. Microbiol. 73, 2762-2764.

[38] Stasiewicz, M. J., Falade, T. D., Mutuma, M., Mutiga, S. K., Harvey, J. J., Fox, G., Pearson, T. C., Muthomi, J. W., and Nelson, R. J. (2017). Multi-spectral kernel sorting to reduce aflatoxins and fumonisins in Kenyan maize. Food Control 78, 203-214.

[39] Stenske, K. A., Smith, J. R., Newman, S. J., Newman, L. B., and Kirk, C. A. (2006). Aflatoxicosis in dogs and dealing with suspected contaminated commercial foods. Journal of the American Veterinary Medical Association 228, 1686-1691.

[40] Stepman, F. (2018). Scaling-up the impact of aflatoxin research in Africa. The role of social sciences. Toxins 10, 136.

[41] Suliman, S., and Abdalla, M. (2013). Presence of aflatoxin M1 in dairy cattle milk in Khartoum StateSudan. Poljoprivreda i Sumarstvo 59, 199.

[42] Unnevehr, L., and Grace, D. (2013). "Aflatoxins: Finding solutions for improved food safety," Intl Food Policy Res Inst.

[43] Williams, J. H., Phillips, T. D., Jolly, P. E., Stiles, J. K., Jolly, C. M., and Aggarwal, D. (2004). Human aflatoxicosis in developing countries: a review of toxicology, exposure, potential health consequences, and interventions. The American journal of clinical nutrition 80, 1106-1122.

[44] Wolde, M. (2017). Effects of aflatoxin contamination of grains in Ethiopia. Int J Agric Sci 7, 1298-308.

[45] Zhang, Y.-J. (2010). Interactions of chemical carcinogens and genetic variation in hepatocellular carcinoma. World journal of hepatology 2, 94.

Citation: Friat Kidanemariam \& Haben Fesseha, "Aflatoxicosis in Animal Products and its Public Health Importance: A Review" International Journal of Research Studies in Microbiology and Biotechnology (IJRSMB), vol. 6, no. 1, pp. 21-29, 2020. Available: DOI: http://dx.doi.org/ 10.20431/2454-9428.0601003

Copyright: (C) 2020 Authors. This is an open-access article distributed under the terms of the Creative Commons Attribution License, which permits unrestricted use, distribution, and reproduction in any medium, provided the original author and source are credited. 